\title{
Bevacizumab (Avastin) Use in Neovascular Glaucoma, and Beyond
}

\author{
Michael Herceg, Robert Noecker \\ UPMC Eye Center, University of Pittsburgh School of Medicine, Eye and Ear Institute, Pittsburgh
}

\section{INTRODUCTION}

The recombinant, full-length, monoclonal IgG1 antibody, bevacizumab (Avastin), binds to all isoforms of vascular endothelial growth factor (VEGF-A). VEGF has been shown to be a potent mediator of both vascular proliferation and increased vascular permeability. Bevacizumab is approved in the United States for intravenous use in the treatment of metastatic colorectal cancer. In ophthalmology, its off-label intraocular and periocular use in the treatment of various neovascular, inflammatory, and neoplastic disorders has become quite popular, and in fact, the standard of care for certain diseases, such as exudative age-related macular degeneration (AMD). As ophthalmologists are becoming increasingly comfortable and impressed with its remarkable efficacy and favorable side effect profile, the number of potential indications and uses continues to increase. ${ }^{1}$

Retinal specialists have encountered success with its use in the treatment of both retinal and choroidal neovascular processes, including AMD, proliferative diabetic retinopathy (PDR), radiation retinopathy, myopic choroidal neovascularization, and retinal neovascularization secondary to vascular occlusion. ${ }^{2}$ In addition, because bevacizumab inhibits the VEGFinduced increase in vascular permeability, it thereby limits the influx of inflammatory mediators and cytokines, and has therefore been used successfully in the treatment of such disorders as refractory cystoid macular edema (CME), diabetic macular edema (without neovascularization), and macular edema associated with vascular occlusions (without neovascularization). ${ }^{3}$ Bevacizumab has even begun to see use in the treatment of various pediatric ocular diseases, such as retinopathy of prematurity, Coat's disease, and choroidal neovascularization associated with Best's disease.

Similarly, glaucoma specialists have started using bevacizumab increasingly in the treatment of neovascular glaucoma (NVG), and to a lesser extent in the management of failing blebs.

Neovascular glaucoma can be classified as a secondary angle closure glaucoma which can be characteristically difficult to control. The disease process goes through several phases, with the early phases being more reversible than the later ones. Most commonly, as a result of retinal ischemia, angiogenic factors diffuse into the aqueous and anterior segment, and interact with the vascular structures where the greatest aqueoustissue contact occurs-namely the iris. These factors provide the stimulus for new vessel, as well as fibrovascular membrane formation. Initially, these membranes can cover the trabecular meshwork causing a secondary open angle glaucoma. As the process continues, the fibrovascular membranes mature and contract, tenting the iris up toward the trabecular meshwork. This initially causes appositional closure of the angle, and eventually permanent peripheral anterior synechiae (PAS) formation. Once enough of the drainage angle is occluded by this synechial closure, a progressive decline in the outflow facility of the eye occurs, and the intraocular pressure (IOP) becomes elevated (often to a significant degree). Thus the formation of a secondary closed angle NVG ensues. This IOP increase is often acute in onset, and to such an extent that it can cause a significant amount of pain to the patient. Other less common causes of NVG are intraocular neoplasm, chronic retinal detachment, and severe intraocular inflammatory disorders.

Iris fluorescein angiographic studies have revealed that the earliest detectable change in iris vascularity in NVG occurs as leakage of fluorescein dye from vessels at the pupillary margin. ${ }^{4}$ This phenomenon has been shown to occur even when clinical slit lamp examination of the iris is normal, and presumably reflects an increased permeability of existing iris vasculature. As the disorder progresses, abnormal new vessels can be detected most often initially at the pupillary margin, and then eventually in the chamber angle. These new vessels typically arise from the iris base as individual fibrovascular trunks, then cross the ciliary body and scleral spur, and reach the trabecular meshwork. Here they branch out and grow circumferentially, and because they possess contractile properties, eventually lead to progressive synechiae formation-and ultimately angle closure.

Medical treatment is centered around aqueous suppression, anti-inflammatory treatment with corticosteroids, and 
cycloplegics in attempt to keep the angle open. Laser treatment of this disorder is aimed largely at retinal ablation via extensive panretinal photocoagulation (PRP). Destroying the peripheral retina decreases the amount of viable retina, and has been proven to inhibit and reverse neovascularization in both the anterior and the posterior segments. This occurs by eliminating ischemic retina, thought to be the source of the release of diffusible substances, such as VEGF, which underlie neovascular process throughout the eye. Secondary treatments are primarily ciliary destructive procedures - most commonly diode laser cyclophotocoagulation (CPC). Surgical approaches include filtering procedures, particularly tube-shunts because of the high rate of failure of trabeculectomies. Retrobulbar ethanol injection and enucleation are unfortunately indicated in a few particularly difficult and unresponsive cases. It is also important to always remember that potentially the most important intervention involved in treating NVG is determining and treating the underlying cause of the formation of neovessels in the first place. This can potentially protect the contralateral eye, as well as prevent more sinister adverse medical events.

Elevated levels of VEGF have been detected in aqueous samples of patients with NVG. ${ }^{5}$ It is postulated that the VEGF initially plays an instrumental role in increased permeability of the existing iris vessels. This increased permeability allows other vasoproliferative mediators and inflammatory cells into the area. Then, as the disease progresses, VEGF provides the stimulus that ultimately leads to new vessel formation. Because of the seemingly central role that VEGF plays in the pathogenesis of NVG, it seems a logical assumption that inhibition of its action via one of the newer anti-VEGF agents could help to slow, halt, or even reverse this process.

Several small case series have been published using bevacizumab as both a primary and an adjunctive therapy to refractory NVG. ${ }^{6-14}$ It has been used via both intracameral and intravitreal routes, with all reporting similar encouraging results. The largest of these studies by Iliev et al, looked at six patients with symptomatic intraocular (IOP) elevation from NVG due to previous retinal vascular occlusions which had been refractory to topical medications. ${ }^{15}$ Patients were given $1.25 \mathrm{mg}$ of intravitreal bevacizumab $(0.05 \mathrm{ml}$ of standard $100 \mathrm{mg} / 4 \mathrm{ml}$ preparations), and PRP was performed as soon as feasible. All cases demonstrated either marked regression or marked complete disappearance of iris neovascularization within 48 hours. In three patients, IOP was well controlled without the need for additional intervention, and three required subsequent diode laser CPC.

There have been several subsequent studies supporting these findings. Grisanti et al demonstrated improvement of clinical iris neovascularization, as well as improvement in iris fluorescein angiography, two days after intracameral injection of $0.04 \mathrm{ml}$ bevacizumab (1 mg). ${ }^{16}$
The effect of bevacizumab alone on the reduction of IOP in neovascular glaucoma remains unclear. Most of the cases reported have used bevacizumab as an adjunct to the more classic NVG treatments, such as PRP and CPC. Theoretically, if given early enough, before permanent synechial closure of the angle has occurred, bevacizumab-induced neovascularization of the angle (NVA) could be reversed, allowing a sufficient degree of open angle to lower IOP without need for cyclodestructive procedures. This would avoid the discomfort and decreased vision that often accompany CPC. This of course, re-emphasizes the importance of careful vigilance for the first signs of iris or angle neovascularization in patients at high risk, such as proliferative diabetic retinopathy, retinal vascular occlusions, and ocular ischemic syndrome. Additionally, aside from potentially lowering the IOP (thereby decreasing the amount of discomfort that can accompany markedly increased pressure), the reduction of NVI can decrease the overall load of intraocular inflammatory mediators, which can also improve patient comfort. Conversely, once permanent synechial closure of the majority of the angle has occurred, avastin alone will not have significant effect on the overall IOP, and alternative interventions, such as CPC, will be needed.

The extent of bevacizumab's long-term efficacy also remains unclear, but is probably quite variable between patients. Currently, the longest reported follow up in the literature extends to at least 10 weeks. Long-term studies will need to be done in order to fully elucidate this.

In our current clinical practice, we routinely administer intravitreal bevacizumab $0.05 \mathrm{ml}$ ( $1 \mathrm{mg}$ ) for NVG, once topical aqueous suppressants, topical steroid anti-inflammatory and atropine 1 percent are no longer sufficient to control IOP. Depending on the extent of IOP elevation and patient discomfort, we will perform concurrent diode CPC. If the patient has not yet received PRP, we also recommend this as soon as feasible. Often either vitreous hemorrhage (from concomitant posterior segment neovascularization) or poor pupillary dilation, preclude adequate visualization for PRP. We have been repeatedly impressed with bevacizumab's ability to dry up neo-vessels in both the anterior and the posterior segments within a few days time, allowing for PRP to be performed.

Many of these patients will still eventually require filtering surgery, for which we typically recommend tube-shunt placement, rather than trabeculectomy, given the high failure rate of these blebs (even in the presence of mitomycin $C$ antimetabolite use). In these patients, the bevacizumab-induced regression of NVI helps to quiet the eye down pre-operatively, thereby reducing intra- and postoperative bleeding, and postoperative inflammation. This, in turn, helps with the eventual long-term success of the filtering device.

In addition to its role in the treatment of NVG, bevacizumab has also been reported for use in needle bleb revision of encapsulated blebs. ${ }^{17,18}$ After trabeculectomy, one of the main 
reasons for failure of the bleb is encapsulation. This capsule forms after vascularization and subsequent influx of mitogenic cytokines. As with other tissues throughout the body, healing of the conjunctiva after surgical trabeculectomy occurs in distinct phases: the acute inflammatory phase, regeneration, repair, and finally contraction. The acute phase occurs within minutes to hours, and involves release of tissue activators at the site of injury which quickly causes clotting in blood vessels adjacent to the injury. Next, in the regeneration phase, replacement of lost cells occurs by mitosis of labile epithelial cells. These new cells undergo restructuring by granulation tissue composed largely of fibroblasts and modified smooth muscle cells, in the repair phase. The repair and regeneration phases last on the order of days to a few weeks. Lastly, over the next intervening months in the contraction phase, the granulation tissue and fibroblasts undergo contraction and shrinkage of the scar. It is during this phase of healing that a capsule can form around the bleb. Throughout this process, cytokines are released that initiate a complex signal cascade, eventually leading to the migration of fibroblasts. These fibroblasts release additional substances which lead not only to encapsulation, but also to scarring of the fistulous tract. VEGF is mitogen specific to vascular endothelial cells, and is closely involved in fibroblast migration and proliferation. The logic behind the use of bevacizumab in this situation is once again blocking the neovascular signal cascade, leading to decrease in the supply of cytokines and fibroblast growth factor (from both the neo-vessels, as well as leakage from pre-existing ones), which in turn decreases fibroblast migration, proliferation, and eventual contraction.

In our clinical practice we have had marginal success with bevacizumab in this setting. Typically, unless the failed encapsulated bleb is particularly vascular, we will initially attempt needling with mitomycin C (MMC $0.04 \mathrm{mg}$ ), prior to attempting needling with bevacizumab. If this initial attempt is unsuccessful, we will then attempt repeat needling with bevacizumab prior to pursuing more aggressive surgical approaches. As in neovascular glaucoma, timing is important. If the vascularization is more prominent and early, an early dose of bevacizumab is indicated. Once a significant amount of subconjunctival fibrosis, scarring, and encapsulation occurs, then there appears to be limited clinical utility to applying the bevacizumab. Likewise, the clinical response can be quite rapid-within days regression of the vessels can be observed. Multiple injections can then be performed over time, depending on the clinical response.

As ophthalmologists' experience and success with the use of intraocular bevacizumab continues to grow, so too do the number of indications and disease states that it is being applied to. Seemingly innumerable disease states share a common foundation centered around the importance of the role of inflammation. This inflammation unavoidably involves the presence of blood vessels and leakage of inflammatory mediators from these vessels. VEGF has been shown to be one of the most powerful stimulants for new fibrovascular tissue growth, as well as increasing permeability of existing vessels. Blocking the effects of VEGF through the use of anti-VEGF antibodies, such as bevacizumab has become a powerful tool in the treatment of many various ocular diseases, including neovascular glaucoma and failing vascularized blebs.

\section{REFERENCES}

1. Geitzenauer W, Michels S, Prager F, Kornek G, Vormittag L, Rosenfeld P, Schmidt-Erfurth U. Early effects of systemic and intravitreal bevacizumab (avastin) therapy for neovascular agerelated macular degeneration. Klin Monatsbl Augenheilkd 2006;223(10):822-7.

2. Lynch SS, Cheng CM. Bevacizumab for neovascular ocular diseases. Ann Pharmacother. 2007;41(4):614-25. Epub 2007;13. Review.

3. Rosenfeld PJ, Fung AE, Puliafito CA. Optical coherence tomography findings after an intravitreal injection of bevacizumab (avastin) for macular edema from central retinal vein occlusion. Ophthalmic Surg Lasers Imaging. 2005;36(4):336-9.

4. Dueker DK. Neovascular Glaucoma. In: Epstein DL, Allingham RR, Schuman JS, (Eds). Chandler and Grant's Glaucoma. Baltimore, MD: Williams and Wilkins, 1997; 309-318.

5. Tripathi RC, Li J, Tripathi BJ, et al. Increased level of vascular endothelial growth factor in aqueous humor of patients with neovascular glaucoma. Ophthalmology 2001;105: 232-7.

6. Yazdani S, Hendi K, Pakravan M. Intravitreal bevacizumab (avastin) injection for neovascular glaucoma. J Glaucoma 2007;16(5):437-9.

7. Bakri SJ, Pulido JS, McCannel CA, Hodge DO, Diehl N, Hillemeier J. Immediate intraocular pressure changes following intravitreal injections of triamcinolone, pegaptanib, and bevacizumab. Eye. 2007 Aug 10; [Epub ahead of print].

8. Chilov MN, Grigg JR, Playfair TJ. Bevacizumab (Avastin) for the treatment of neovascular glaucoma. Clin Experiment Ophthalmol. 2007;35(5):494-6.

9. Batioglu F, Astam N, Ozmert E. Rapid improvement of retinal and iris neovascularization after a single intravitreal bevacizumab injection in a patient with central retinal vein occlusion and neovascular glaucoma. Int Ophthalmol. 2007 Jul 4; [Epub ahead of print].

10. Vatavuk Z, Bencic G, Mandic Z. Intravitreal bevacizumab for neovascular glaucoma following central retinal artery occlusion. Eur J Ophthalmol. 2007;17(2):269-71.

11. Mason JO 3rd, Albert MA Jr, Mays A, Vail R. Regression of neovascular iris vessels by intravitreal injection of bevacizumab. Retina 2006;26(7):839-41. 
12. Silva Paula J, Jorge R, Alves Costa R, Rodrigues Mde L, Scott IU. Short-term results of intravitreal bevacizumab (Avastin) on anterior segment neovascularization in neovascular glaucoma. Acta Ophthalmol Scand. 2006;84(4):556-7.

13. Kahook MY, Schuman JS, Noecker RJ. Intravitreal bevacizumab in a patient with neovascular glaucoma. Ophthalmic Surg Lasers Imaging 2006;37(2):144-6.

14. Davidorf FH, Mouser JG, Derick RJ. Rapid improvement of rubeosis iridis from a single bevacizumab (Avastin) injection. Retina 2006;26(3):354-6.

15. Iliev ME, Domig D, Wolf-Schnurrbursch U, et al. Intravitreal bevacizumab (Avastin) in the treatment of neovascular glaucoma. Am J Ophthalmo. 2006;142(6): 1054-6.

16. Grisanti S, Biester $S$, Peters $S$, et al. Intracameral bevacizumab for iris rubeosis. Am J Ophthalmo 2006;142(1): 158-60.

17. Kahook MY, Schuman JS, Noecker RJ. Needle bleb revision of encapsulated filtering bleb with bevacizumab. Ophthalmic Surg Laser Imaging 2006;37(2):148-50.
18. Jonas JB, Spandau UH, Schlichtenbrede F. Intravitreal bevacizumab for filtering surgery. Ophthalmic Res 2007; 39(2):121-2.

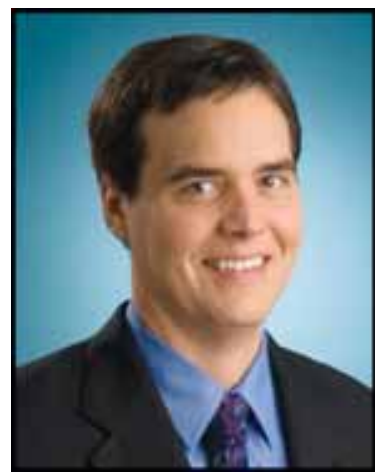

Dr Robert Noecker (noeckerrj@UPMC.EDU)

"The ideals which have lighted my way, and time after time have given me new courage to face life cheerfully, have been kindness, beauty and truth”

— Albert Einstein 